\title{
The role of apoptosis in pulmonary fibrosis
}

\author{
B.D. Uhal
}

ABSTRACT: Apoptosis has been defined as "gene-directed cellular self-destruction" and is an active process that is tightly regulated by a number of gene products, which promote or block cell death. Apoptotic death can be triggered by a wide variety of stimuli and, importantly, not all cells necessarily undergo apoptosis in response to the same stimulus. Abnormal regulation of apoptosis has been implicated in a wide range of diseases and approaches to modifying apoptosis represent important future therapeutic strategies.

Idiopathic pulmonary fibrosis (IPF) is a progressive and relentless disease involving scarring of the lung, which has been recognised as the most lethal interstitial lung disease. In the lungs of IPF patients, increased epithelial apoptosis, together with decreased apoptosis of myofibroblasts, represents persistent findings (particularly in areas of collagen deposition) supporting an interaction between altered apoptosis and the pathogenesis of the disease.

Data from human tissues and animal models are refining current knowledge of the processes involved in this pathogenesis. This has challenged the dogma that IPF is purely a disease of unresolved inflammation by emphasising the central roles played by the alveolar epithelial cell and myofibroblasts and, as part of that role, the importance of altered apoptosis.

Evidence suggests blockade of epithelial cell apoptosis can prevent subsequent collagen deposition, and induction of myofibroblast apoptosis, at least theoretically, would be expected to resolve ongoing fibrosis. These two concepts raise the prospect of therapeutic intervention aimed at modifying apoptosis and, thus, fibrosis in idiopathic pulmonary fibrosis.

KEYWORDS: Angiotensin, epithelium, lung, myofibroblasts

A poptosis is a highly organised physiological event that plays a pivotal role in a range of normal developmental and homeostatic processes. It has been defined as "gene-directed cellular self-destruction" and is tightly regulated by a number of gene products that promote or block cell death. Apoptosis is an active process that requires both gene expression and cell-cycle progression. A wide range of stimuli have been shown to induce apoptotic cell death, although their ability to do so varies in different cell types and under different circumstances, and certain cells may show variability in their resistance or liability to apoptosis in response to the same stimuli. This has important implications in the pathogenesis of certain diseases and, potentially, for their treatment. One such disease is idiopathic pulmonary fibrosis (IPF), a distinctive type of chronic fibrosing interstitial pneumonia of unknown cause, which is associated with a histological pattern of usual interstitial pneumonia.

Epithelial apoptosis is a persistent finding in lung tissue from patients with IPF (fig. 1) [1, 2] and in murine models of bleomycin (BLM)-induced pulmonary fibrosis $[3,4]$. Experiments using in situ labelling techniques have demonstrated numerous apoptotic epithelial cells in lung tissue from patients with IPF, which are associated with areas of myofibroblast activity and abnormal alveoli $[2,5]$. In addition, increased expression of pro-apoptotic proteins and decreased expression of anti-apoptotic proteins, both within epithelial cells, has also been reported [1]. Conversely, more recent evidence documents resistance to apoptosis in lung myofibroblasts, the key cell type believed to be responsible for collagen deposition in IPF. Although the natural history and the pathogenic mechanisms of IPF are unknown, there is increasing evidence that apoptosis is increased in alveolar epithelial cells (AECs) but decreased in myofibroblasts in IPF, and that these mechanisms may be pivotal to the underlying pathogenesis.

Recently, therefore, the long-prevailing hypothesis that IPF arises from an unresolved inflammatory process where AECs are destroyed by the inflammation has been challenged. Instead, a model has been proposed in which IPF results from multiple cycles of epithelial cell injury and activation that provoke the migration, proliferation and activation of mesenchymal cells with the
CORRESPONDENCE

B.D. Uhal

Dept of Physiology

Michigan State University

3197 BPS Building

East Lansing

MI 48824

USA

Fax: 15173555125

E-mail: uhal@msu.edu

STATEMENT OF INTEREST

B.D. Uhal has received consultancy fees and travel funds from Actelion, Ltd and Actelion Pharmaceuticals, Ltd. 

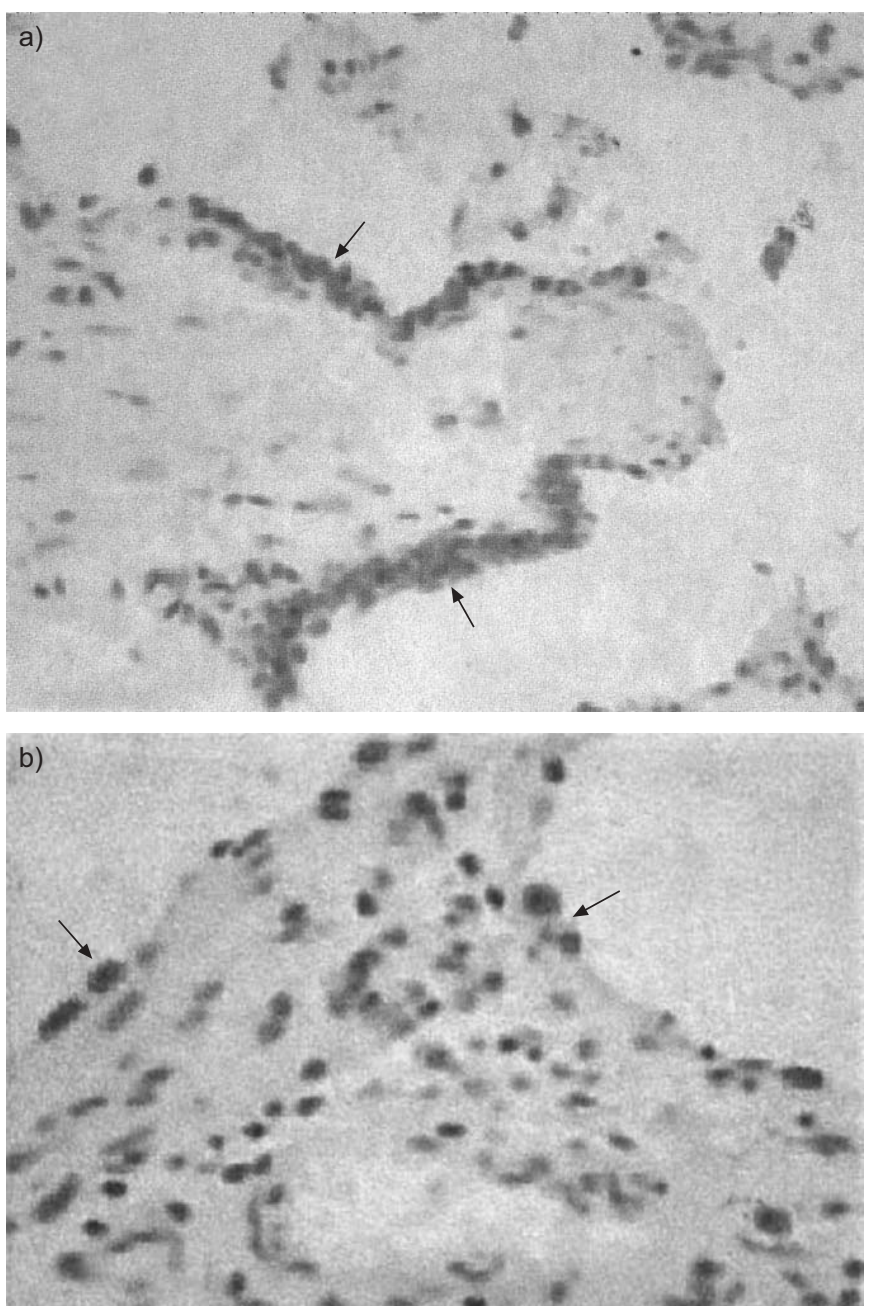

FIGURE 1. $a$ and b) Representative staining results of terminal deoxynucleotide transferase-mediated deoxyuridine triphosphate-biotin nick end-labelling in paraffin-embedded lung tissue from patients with idiopathic pulmonary fibrosis. The microscopic areas of epithelial cell dropout and increased apoptosis immediately overlying fibroblastic foci that are virtually devoid of labelling, consistent with decreased apoptosis of myofibroblasts, are shown by arrows. Reproduced from [1] with permission from the publisher.

formation of active and persistent fibroblastic/myofibroblastic foci; a process which mirrors abnormal wound repair [6].

\section{MYOFIBROBLAST APOPTOSIS IN IPF}

In skin wound healing models, apoptosis has been shown to be a normal mechanism for removal of the excess myofibroblasts. In granulation tissue, myofibroblasts develop ultrastructural and biochemical features of smooth muscle (SM) cells, including the expression of $\alpha$-SM actin ( $\alpha$-SMA) [7]. In this situation, when granulation tissue resolves, myofibroblasts containing $\alpha$-SMA are removed by apoptosis as part of the normal healing process. In contrast, myofibroblasts expressing $\alpha$-SMA persist in regions where there is excessive scarring and in fibrotic conditions [7]. As skin wounds heal, there is an increase in in situ end labelling of fragmented DNA, a marker of apoptosis, in both myofibroblasts and vascular cells. This apoptosis is thought to be due to the combined effects of reduced growth factor expression, increased extracellular matrix turnover and nitric oxide generation [8].

If markers for apoptosis are examined in lung biopsies from patients with IPF, heavy labelling is only seen in the epithelium overlying myofibroblast foci, not in the myofibroblasts themselves, suggesting resistance to apoptosis in these cells. This resistance to apoptosis has been demonstrated in vitro, where myofibroblasts isolated from fibrotic lung were found to be more resistant to Fas ligand-induced apoptosis than normal controls [9]. One mechanism for this reduced susceptibility may be lower expression of cell surface Fas, together with higher levels of apoptosis-inhibiting soluble Fas by these cells [10]. In addition, interleukin (IL)-6, a cytokine known to be involved in repair and remodelling, enhances Fasinduced apoptosis and expression of the apoptosis-promoting protein Bax in normal fibroblasts, but conversely inhibits apoptosis and induces expression of the anti-apoptopic protein Bcl-2 in fibroblasts from patients with IPF [11]. These studies suggest that mesenchymal cells in IPF develop an apoptosisresistant phenotype, which contributes to persistent or progressive fibrosis.

A number of factors have been shown to induce apoptosis in lung fibroblasts, either in culture or in animal models (table 1); these include ILs (including IL-1 $\beta$ ), hepatocyte growth factor (HGF) and disruption of integrin $\alpha 2 \beta 1$ attachment. Factors known to confer resistance to apoptosis in the same cells (table 2) include transforming growth factor (TGF)- $\beta 1$, thrombin, insulin-like growth factor (IGF)-1 and FIZZ (found in inflammatory zone)-1. Therefore, potential therapeutic targets in IPF may involve the administration of some inducers of myofibroblast apoptosis or promotion of their mediators. Alternatively, potential therapies might be based on the blockade of endogenous factors that confer resistance to apoptosis (or their mediators), provided this can be achieved in a cell type-specific manner.

\section{EPITHELIAL CELL APOPTOSIS IN IPF}

As previously mentioned, in situ labelling methods have demonstrated numerous apoptotic cells in lung tissue from patients with IPF [2, 5], and the affected cell type appears to be type-II pneumocytes [5]. Type-II pneumocytes, but not type-I cells, are capable of undergoing mitosis and transformation into type-I pneumocytes [21]. Type-II pneumocytes are responsible for the synthesis, storage and secretion of surfactant, which means that their loss is particularly significant. Apoptotic AECs have been found in areas of normal alveoli, suggesting that apoptosis in AECs is a primary event in the pathogenesis of IPF prior to the onset of fibrosis [5]. Markers of apoptosis have also been reported in bronchiolar cells and AECs associated with regions of the largest myofibroblast activity and collagen accumulation in lung biopsy specimens from patients with IPF [2].

There is a variety of mechanisms by which epithelial apoptosis might be important in the pathogenesis of a fibrotic response. The epithelium of any organ has important antifibrotic functions and in the lung, epithelial cells are involved in the production of mediators that inhibit fibroblast proliferation (e.g. prostaglandin $\left(\mathrm{PG} \mathrm{E}_{2}\right.$ ) and degrade interstitial fibrin and collagen (e.g. plasminogen activators and metalloproteinase). 
TABLE 1 Inducers of apoptosis in lung fibroblasts

\begin{tabular}{|c|c|c|}
\hline Inducer & Mediators & Ref. \\
\hline IL-1 $\beta$ & Inducible nitric oxide synthase & [12] \\
\hline Hepatocyte growth factor & MMP-2/-9, focal adhesion kinase dephosphorylation & [14] \\
\hline Soluble fibronectin peptides ${ }^{\#}$ & Focal adhesion kinase (125 kDa form; pp125FAK) proteolysis & {$[15]$} \\
\hline $\begin{array}{l}\text { Contraction of collagen matrix or disruption of integrin } \alpha 2 \beta 1 \\
\text { attachment (anoikis) }\end{array}$ & Akt, focal adhesion kinase dephosphorylation & {$[16,17]$} \\
\hline
\end{tabular}

In addition, intact lung epithelial cells provide a physical barrier which may prevent pro-fibrotic molecules, e.g. cytokines from activated alveolar macrophages, from reaching underlying tissue. Therefore, it is possible to envision, at least theoretically, that if epithelial cells are removed by increased apoptosis there could be a collapse of barrier function, which allows pro-fibrotic cytokines from activated alveolar macrophages access to the underlying fibroblasts. At the same time, a reduction in the production of growth inhibitors (e.g. $\left.\mathrm{PGE}_{2}\right)$, less fibrinolysis and less matrix metalloproteinase production could lead to a pro-fibrotic microenvironment.

If this theory is correct, blocking epithelial apoptosis in IPF may be expected to block subsequent fibrosis. This has been demonstrated in a number of studies $[4,22,23]$. The broadspectrum caspase inhibitor Z-Val-Ala-Asp-fluoromethylketone has been shown to inhibit BLM-induced apoptosis of alveolar and airway wall cells in a rat model of BLM-induced lung fibrosis, and prevent subsequent collagen deposition [22]. Further evidence of the importance of apoptosis in the development of pulmonary fibrosis in a BLM model originates from recent work by BUDINGER et al. [23], who demonstrated that mice lacking the pro-apoptotic Bcl-2 family member Bid showed significantly less pulmonary fibrosis in response to BLM compared with controls, despite similar levels of inflammation, lung injury and active pro-fibrotic TGF- $\beta 1$.

These studies show that different strategies can be used successfully to block AEC apoptosis and reduce collagen deposition, at least in animal models, raising the possibility of therapeutic control of lung fibrosis by targeting apoptosis. Mediators involved in AEC apoptosis in response to a number of inducers have been identified previously (table 3) and include caspases, members of the Bcl family and angiotensin (ANG) receptors. Therefore, potential therapeutic strategies for IPF include the inhibition of expression or blockade of these inducers or their mediators; however, these must also be performed in a cell type-specific manner that will not diminish the desirable apoptosis of myofibroblasts discussed previously. A number of AEC apoptosis blockers have been demonstrated to reduce both apoptosis and lung collagen deposition in mice or rat models of IPF (table 3), among which the most potentially interesting are the ANG-1 receptor blockers.

\section{ANG AND PULMONARY FIBROSIS}

In IPF, the survival of AECs can be seen as a battle between cell death, cell proliferation and repair. The high expression of p21 in these cells probably reflects attempts to rescue the epithelium from apoptosis and promote this repair. Reactive epithelium such as this produces a number of cytokines and factors that are known to have pro-apoptotic and pro-fibrotic activity, among them ANGII.

Although capillary endothelial cells are considered to be the most important producers of circulating ANGII, autocrine synthesis of ANGII by AECs can be induced by a number of factors. Activation of Fas stimulates AECs to synthesise ANGII and the autocrine synthesis of ANGII has been found to be required for the induction of apoptosis by Fas in AECs [27]. This Fas-induced apoptosis could be abrogated by ANG receptor

TABLE 2 Factors that confer resistance to apoptosis in lung myofibroblasts/fibroblasts

\begin{tabular}{|c|c|c|c|}
\hline IL-6 & Reduces FasL induction of apoptosis & STAT-3, Bcl-2 & [11] \\
\hline Thrombin & Blocks FasL induction & PAR1, protein kinase $C-\varepsilon, p 21$ & [18] \\
\hline IGF-1 & Blocks induction by growth factor withdrawal & Akt, ERK phosphorylation & [19] \\
\hline FIZZ-1 ${ }^{\#}$ & Blocks TNF- $\alpha$ induction & ERK1/-2 phosphorylation & [20] \\
\hline
\end{tabular}

IL: interleukin; TGF: transforming growth factor; IGF: insulin-like growth factor; Akt: protein kinase B; FasL: Fas ligand; STAT: signal transducer and activator of transcription; iNOS: inducibile nitric oxide synthase; PAR: proteinase-activated receptor; FIZZ: found in inflammatory zone; TNF: tumour necrosis factor; ERK: extracellular signal-related kinase. ${ }^{\#}$ : e.g. resistin-like molecule- $\alpha$. 
TABLE 3 Inducers and blockers of apoptosis in lung epithelial cells

\begin{tabular}{|c|c|c|c|}
\hline Inducer & Mediators & Blocker & Ref. \\
\hline Bleomycin, amiodarone & Caspases, Bcl, AT1 receptor & Caspase inhibitors ${ }^{\#}$, losartan ${ }^{\#}$ & {$[22,24,25]$} \\
\hline TNF- $\alpha$ & Caspases, Bcl, ANGII & P21 overexpression ${ }^{\#}$, captopril ${ }^{\#}$, lisinopril & {$[28,29]$} \\
\hline TGF- $\beta 1$ & Caspases, EGR & caspase inhibitors ${ }^{\#}$ & {$[30]$} \\
\hline
\end{tabular}

antagonists or other blockers of ANGII function. The same requirement for ANGII production applies to the apoptosis of AECs in response to tumour necrosis factor- $\alpha$, which is also blocked by agents that abrogate ANGII function [29]. Subsequently, several studies have shown that a number of inducers of apoptosis, including BLM [31] and amiodarone [24, 25], can induce expression of angiotensinogen (AGT) messenger RNA (mRNA) and that apoptosis can be abrogated by blocking ANGII binding to the ANGII type-I receptor subtype (AT1R).

In comparison to normal lung, the expression of AGT mRNA and protein is increased by $\sim 20$-fold and four-fold, respectively, in lung tissue from patients with IPF. AGT mRNA and protein have been observed to co-localise with markers of apoptosis, which is suggestive of AGT expression by apoptotic epithelia in situ [32]. In addition, although AGT protein and mRNA also co-localised to myofibroblast foci detected by $\alpha$ SMA monoclonal antibody, AGT mRNA was not detected in SM, which suggests that apoptotic AEC and myofibroblasts constitute key sources of locally derived AGT peptides in the IPF lung. This has implications for local fibrosis and collagen deposition as ANGII has been shown to be mitogenic for lung fibroblasts [33] and to stimulate TGF- $\beta 1$ and pro-collagen synthesis [34, 35].

Data from animal models support the importance of ANGII as an important mediator of fibrosis in IPF. ANG receptor knockout mice do not accumulate collagen in response to BLM (fig. 2) [3] and treatment with the AT1R antagonists, i.e. candesartan (fig. 3) [36] or lorsartan [37], reduces fibrosis in the BLM model. Recently it has also been shown that blockade of local pulmonary ANGII synthesis by intratracheal administration of antisense oligonucleotides against AGT mRNA attenuates apoptosis of AECs and reduces BLM-induced pulmonary fibrosis in rats [38].

The notion that similar mechanisms involving AGT gene expression might play a role in human IPF is supported by the recent findings that IPF patients possessing the AA genotype of the G-6A polymorphism of AGT have faster disease progression, as assessed by widening of the alveolar-arterial oxygen gradient, than patients with the GA or GG genotype [39]. In studies of liver fibrosis, the same AA genotype of AGT was associated with significantly more severe fibrosis after hepatitis $C$ infection, particularly when found together with TGF- $\beta 1$ polymorphisms [40].

Taken together, these findings suggest that ANGII is an important mediator in the apoptosis of AECs during lung fibrogenesis, regardless of the initiating stimulus, and that therapeutic control of AEC apoptosis is feasible through pharmacological manipulation of the local renin-ANG system.

\section{THERAPEUTIC MANIPULATION OF APOPTOSIS}

In contrast to necrosis or "accidental" cell death, apoptosis is an active form of cell death, requiring the activation of specific enzymes and signalling pathways, which offers the potential for pharmacological manipulation. Possibly the most important consideration for therapeutic manipulation of apoptosis is the rapid kinetics of the process relative to cell proliferation, which implies that the manipulation of apoptosis could be viewed as having an even greater potential for the alteration of cell population size than does control over cell division.

The biggest potential problem for therapeutic manipulation is cell-type specificity, that is, the ability to block apoptosis in some cells while not interfering with the normal control of other cells. In IPF this means blocking epithelial apoptosis while at the same time inducing apoptosis in myofibroblasts. In this respect, two interesting candidates for therapy are HGF and, as previously discussed, AT1R antagonists.

HGF is the ligand for c-Met, a receptor tyrosine kinase that is shown to be overexpressed and mutated in a variety of malignancies. It was originally identified and cloned as a potent mitogen for hepatocytes and has since been shown to

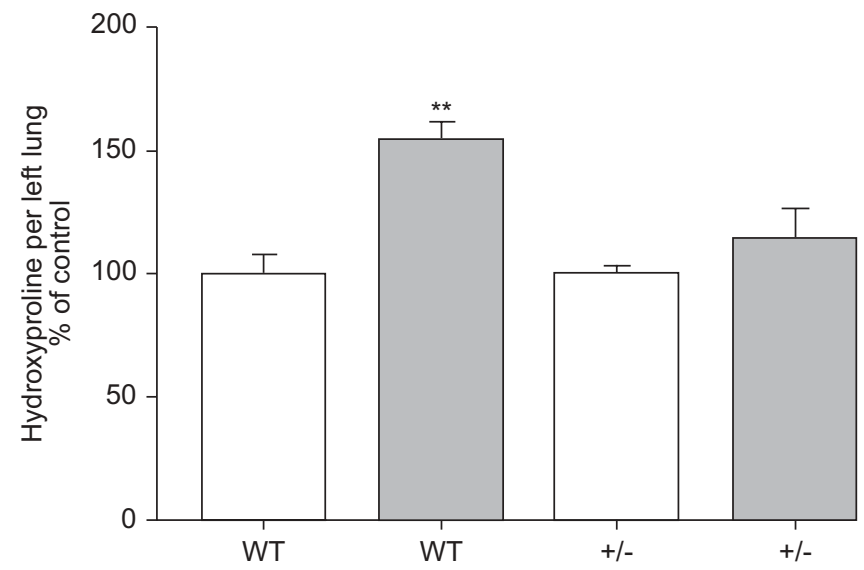

FIGURE 2. Reduced fibrosis in response to bleomycin (BLM) in angiotensin I type-1 receptor knockout mice. $\square$ : no BLM added; $\square$ : BLM added. WT: wild-type mice; +/-: heterozygous AT1a knockout animals. **: $p<0.01$. Reproduced from [3] with permission from the publisher. 


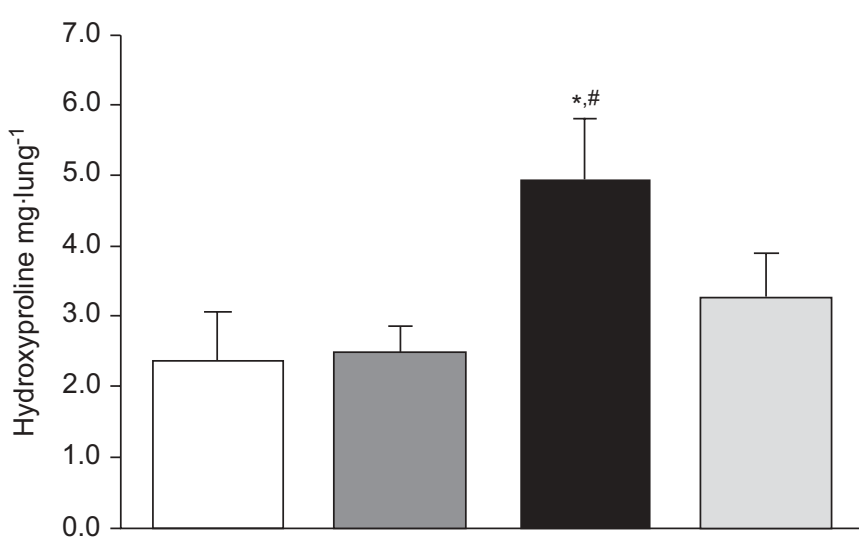

FIGURE 3. Antifibrotic effects observed following antagonism of angiotensin ॥ type-1 receptors in a rat model of bleomycin (BLM)-induced pulmonary fibrosis. $\square$ : saline alone; $\square$ : saline plus candesartan $\left(10 \mathrm{mg} \cdot \mathrm{kg}^{-1} \cdot \mathrm{day}^{-1}\right)$; $\mathbf{\square}$ : BLM alone; II: BLM plus candesartan $\left(10 \mathrm{mg} \cdot \mathrm{kg}^{-1} \cdot \mathrm{day}^{-1}\right) .{ }^{*}: \mathrm{p}<0.05$ versus saline alone; \#: $p<0.05$ versus BLM plus candesartan. Reproduced from [36] with permission from the publisher.

have mitogenic, motogenic and morphogenic activities for a wide variety of cells. HGF is known to have an anti-apoptotic effect on epithelial cells, enhance apoptosis in myofibroblasts [41] and is involved in the local regulation of fibrinolysis and coagulation [42]. Clinically, levels of HGF have been found to be elevated in both serum [43] and bronchoalveolar lavage fluid [44] of patients with either IPF or collagen vascular disease-associated pulmonary fibrosis. In the BLM-induced lung injury mouse model, administration of HGF reduces fibrosis and morphological changes [45]. This effect can be observed if HGF is co-administered with BLM or if HGF is administered 2 weeks after instillation of BLM, which suggests that HGF may reverse some of the BLM-induced fibrotic changes. Delayed administration of intratracheal HGF has been shown to not only reduce fibrosis in this model, but also to increase bronchial epithelial and alveolar epithelial proliferation [46]. In addition, several studies have shown that gene transfer of HGF increases lung epithelial cell proliferation and reduces apoptosis in BLM-induced fibrosis, suggesting increased alveolar epithelial repair, and also decreases levels of TGF- $\beta[47,48]$.

The profibrotic potential of ANGII is believed to be mediated by: upregulation of collagen gene expression in lung fibroblasts [33]; induction of apoptosis in alveolar epithelial cells [3]; and other profibrotic actions [49]. A recent retrospective review showed no benefit of ANG-converting enzyme (ACE) inhibitors on survival in patients with IPF [50], which may seem to argue against the potential efficacy of ANG system antagonists in this population. However, ACE inhibitors would not necessarily be expected to be as effective as specific AT1R antagonists, as ACE is not the predominant enzyme in the local ANG generating system and there are at least five other enzymes that can directly convert ATI or AGT to ANGII that are not inhibited by ACE inhibitors. Therefore, although evidence suggests that ACE inhibitors do not have a positive effect in IPF, AT1R antagonists may prove more beneficial. Administration of an AT1R antagonist has been shown to significantly attenuate pulmonary fibrosis in vivo [22, 34, 36, 51-53] (fig. 3) and to inhibit Fas-induced apoptosis in human lung cells in vitro [54]. Indeed, a small clinical trial of IPF patients [55] showed that the AT1R antagonist losartan elicited a positive effect on forced vital capacity when administered to IPF patients for $1 \mathrm{yr}$.

Taken together, these results and those from experimental models indicate that hepatocyte growth factor and angiotensin II type-I receptor subtype antagonists may represent promising candidates as potential therapies for idiopathic pulmonary fibrosis. However, further investigations need to be carried out to explain the apparently paradoxical apoptosis in idiopathic pulmonary fibrosis, in which alveolar epithelial cells appear susceptible to apoptosis while myofibroblasts display apparent resistance to this process in the same tissue environment. Understanding the biochemical and molecular basis for these differences may lead to the development of more specific and targeted therapies for idiopathic pulmonary fibrosis.

\section{REFERENCES}

1 Plataki M, Koutsopoulos AV, Darivianaki K, Delides G, Siafakas NM, Bouros D. Expression of apoptotic and antiapoptotic markers in epithelial cells in idiopathic pulmonary fibrosis. Chest 2005; 127: 266-274.

2 Uhal BD, Joshi I, Hughes WF, Ramos C, Pardo A, Selman M. Alveolar epithelial cell death adjacent to underlying myofibroblasts in advanced fibrotic human lung. Am J Physiol Lung Cell Mol Physiol 1998; 275: L1192-1199.

3 Li X, Rayford H, Uhal BD. Essential roles for angiotensin receptor AT1a in bleomycin-induced apoptosis and lung fibrosis in mice. Am J Pathol 2003; 163: 2523-2530.

4 Hagimoto N, Kuwano K, Nomoto Y, Kunitake R, Hara N. Apoptosis and expression of FAS/FAS ligand mRNA in bleomycin-induced pulmonary fibrosis in mice. Am J Respir Cell Mol Biol 1997; 16: 91-101.

5 Barbas-Filho JV, Ferreira MA, Sesso A, Kairalla RA, Carvalho CR, Capelozzi VL. Evidence of type II pneumocyte apoptosis in the pathogenesis of idiopathic pulmonary fibrosis (IFP)/usual interstitial pneumonia (UIP). J Clin Pathol 2001; 54: 132-138.

6 Selman M, King TE, Pardo A. Idiopathic pulmonary fibrosis: prevailing and evolving hypotheses about its pathogenesis and implications for therapy. Ann Intern Med 2001; 134: 136-151.

7 Desmoulière A. Factors influencing myofibroblast differentiation during wound healing and fibrosis. Cell Biol Int 1995; 19: 471-476.

8 Darby IA, Bisucci T, Pittet B, Garbin S, Gabbiani G, Desmouliere A. Skin flap-induced regression of granulation tissue correlates with reduced growth factor and increased metalloproteinase expression. J Pathol 2002; 197: 117-127.

9 Moodley YP, Caterina P, Scaffidi AK, et al. Comparison of the morphological and biochemical changes in normal human lung fibroblasts and fibroblasts derived from lungs of patients with idiopathic pulmonary fibrosis during FasL-induced apoptosis. J Pathol 2004; 202: 486-495.

10 Bühling F, Wille A, Röcken C, et al. Altered expression of membrane-bound and soluble CD95/Fas contributes to the 
resistance of fibrotic lung fibroblasts to FasL induced apoptosis. Respir Res 2005; 6: 37.

11 Moodley YP, Misso NL, Scaffidi AK, et al. Inverse effects of interleukin-6 on apoptosis of fibroblasts from pulmonary fibrosis and normal lungs. Am J Respir Cell Mol Biol 2003; 29: 490-498.

12 Zhang HY, Phan SH. Inhibition of myofibroblast apoptosis by transforming growth factor $\beta 1$. Am J Respir Cell Mol Biol 1999; 21: 658-665.

13 Ramos C, Montaño M, Becerril C, et al. Acidic fibroblast growth factor decreases $\alpha$-smooth muscle actin expression and induces apoptosis in human normal lung fibroblasts. Am J Physiol Lung Cell Mol Physiol 2006; 291: L871-L879.

14 Mizuno S, Matsumoto K, Li MY, Nakamura T. HGF reduces advancing lung fibrosis in mice: a potential role for MMP-dependent myofibroblast apoptosis. FASEB J 2005; 19: 580-582.

15 Hadden HL, Henke CA. Induction of lung fibroblast apoptosis by soluble fibronectin peptides. Am J Respir Crit Care Med 2000; 162: 1553-1560.

16 Nho RS, Xia H, Kahm J, Kleidon J, Diebold D, Henke CA. Role of integrin-linked kinase in regulating phosphorylation of Akt and fibroblast survival in type I collagen matrices through a $\beta 1$ integrin viability signaling pathway. J Biol Chem 2005; 280: 26630-26639.

17 Tian B, Lessan K, Kahm J, Kleidon J, Henke C. $\beta 1$ integrin regulates fibroblast viability during collagen matrix contraction through a phosphatidylinositol 3-kinase/Akt/ protein kinase B signaling pathway. J Biol Chem 2002; 277: 24667-24675.

18 Bogatkevich GS, Gustilo E, Oates JC, et al. Distinct PKC isoforms mediate cell survival and DNA synthesis in thrombin-induced myofibroblasts. Am J Physiol Lung Cell Mol Physiol 2005; 288: L190-L201.

19 Wynes MW, Frankel SK, Riches DW. IL-4-induced macrophage-derived IGF-I protects myofibroblasts from apoptosis following growth factor withdrawal. J Leukoc Biol 2004; 76: 1019-1027.

20 Chung MJ, Liu T, Ullenbruch M, Phan SH. Antiapoptotic effect of found in inflammatory zone (FIZZ)1 on mouse lung fibroblasts. J Pathol 2007; 212: 180-187.

21 Adamson YR, Bowden DH. The type 2 cell as progenitor of alveolar epithelial regeneration. A cytodynamic study in mice after exposition to oxygen. Lab Invest 1974; 30: 35-42.

22 Wang R, Ibarra-Sunga O, Pick R, Uhal BD. Abrogation of bleomycin-induced epithelial apoptosis and lung fibrosis by captopril or by a caspase inhibitor. Am J Physiol Lung Cell Mol Physiol 2000; 279: L143-L151.

23 Budinger GR, Mutlu GM, Eisenbart J, et al. Proapoptotic Bid is required for pulmonary fibrosis. Proc Natl Acad Sci USA 2006; 103: 4604-4609.

24 Bargout R, Jankov A, Dincer E, et al. Amiodarone induces apoptosis of human and rat alveolar epithelial cells in vitro. Am J Physiol Lung Cell Mol Physiol 2000; 278: L1039-L1044.

25 Uhal BD, Wang R, Laukka J, Zhuang J, Soledad-Conrad V, Filippatos G. Inhibition of amiodarone-induced lung fibrosis but not alveolitis by angiotensin system antagonists. Pharmacol Toxicol 2003; 92: 81-87.

26 Okada M, Sugita K, Inukai T, et al. Hepatocyte growth factor protects small airway epithelial cells from apoptosis induced by tumor necrosis factor- $\alpha$ or oxidative stress. Pediatr Res 2004; 56: 336-344.

27 Wang, R, Zagariya A, Ibarra-Sunga O, Ang E, Uhal BD. Fas-induced apoptosis of alveolar epithelial cells requires ANG II generation and receptor interaction. Am J Physiol Lung Cell Mol Physiol 1999; 277: L1245-L1250.

28 Inoshima I, Kuwano K, Hamada N, et al. Induction of CDK inhibitor p21 gene as a new therapeutic strategy against pulmonary fibrosis. Am J Physiol Lung Cell Mol Physiol 2004; 286: L727-L733.

29 Wang R, Alam G, Zagariya A, et al. Apoptosis of lung epithelial cells in response to TNF- $\alpha$ requires angiotensin II generation de novo. J Cell Physiol 2000; 185: 253-259.

30 Lee CG, Kang HR, Homer RJ, Chupp G, Elias JA. Transgenic modeling of transforming growth factor- $\beta 1$ : role of apoptosis in fibrosis and alveolar remodeling. Proc Am Thorac Soc 2006; 3: 418-423.

31 Li X, Zhang H, Soledad-Conrad V, Zhuang J, Uhal BD. Bleomycin-induced apoptosis of alveolar epithelial cells requires angiotensin synthesis de novo. Am J Physiol Lung Cell Mol Physiol 2003; 284: L501-L507.

32 Li X, Molina-Molina M, Abdul-Hafez A, et al. Extravascular sources of lung angiotensin peptide synthesis in idiopathic pulmonary fibrosis. Am J Physiol Lung Cell Mol Physiol 2006; 291: L887-L895.

33 Marshall RP, McAnulty RJ, Laurent GJ. Angiotensin II is mitogenic for human lung fibroblasts via activation of the type 1 receptor. Am J Respir Crit Care Med 2000; 161: 1999-2004.

34 Marshall RP, Gohlke P, Chambers RC, et al. Angiotensin II and the fibroproliferative response to acute lung injury. Am J Physiol Lung Cell Mol Physiol 2004; 286: L156-L164.

35 Uhal BD, Kim JK, Li X, Molina-Molina M. AngiotensinTGF- $\beta 1$ crosstalk in human idiopathic pulmonary fibrosis: autocrine mechanisms in myofibroblasts and macrophages. Curr Pharm Des 2007; 13: 1247-1256.

36 Otsuka M, Takahashi H, Shiratori M, Chiba H, Abe S. Reduction of bleomycin induced lung fibrosis by candesartan cilexetil, an angiotensin II type 1 receptor antagonist. Thorax 2004; 59: 31-38.

37 Yao HW, Zhu JP, Zhao MH, Lu Y. Losartan attenuates bleomycin-induced pulmonary fibrosis in rats. Respiration 2006; 73: 236-242.

38 Li X, Zhuang J, Rayford H, Zhang H, Shu R, Uhal BD. Attenuation of bleomycin-induced pulmonary fibrosis by intratracheal administration of antisense oligonucleotides against angiotensinogen mRNA. Curr Pharm Des 2007; 13: 1257-1268.

39 Molina-Molina M, Xaubet A, Li X, et al. Angiotensinogen gene G-6A polymorphism influences idiopathic pulmonary fibrosis in disease progression. Eur Respir J 2008; [Epub ahead of print PMID: 18508830].

40 Powell EE, Edwards-Smith CJ, Hay JL, et al. Host genetic factors influence disease progression in chronic hepatitis $\mathrm{C}$. Hepatology 2000; 31: 828-833.

41 Kim WH, Matsumoto K, Bessho K, Nakamura T. Growth inhibition and apoptosis in liver myofibroblasts promoted by hepatocyte growth factor leads to resolution from liver cirrhosis. Am J Pathol 2005; 166: 1017-1028.

42 Wojta J, Nakamura T, Fabry A, et al. Hepatocyte growth factor stimulates expression of plasminogen activator 
inhibitor type 1 and tissue factor in HepG2 cells. Blood 1994; 84: 151-157.

43 Yamanouchi H, Fujita J, Yoshinouchi T, et al. Measurement of hepatocyte growth factor in serum and bronchoalveolar lavage fluid in patients with pulmonary fibrosis. Respir Med 1998; 92: 273-278.

44 Sakai T, Satoh K, Matsushima K, et al. Hepatocyte growth factor in bronchoalveolar lavage fluids and cells in patients with inflammatory chest diseases of the lower respiratory tract: detection by RIA and in situ hybridization. Am J Respir Cell Mol Biol 1997; 16: 388-397.

45 Yaekashiwa M, Nakayama S, Ohnuma K, et al. Simultaneous or delayed administration of hepatocyte growth factor equally represses the fibrotic changes in murine lung injury induced by bleomycin. A morphologic study. Am J Respir Crit Care Med 1997; 156: 1937-1944.

46 Dohi M, Hasegawa T, Yamamoto K, Marshall B. Hepatocyte growth factor attenuates collagen accumulation in murine model of pulmonary fibrosis. Am J Respir Crit Care Med 2000; 162: 2302-2307.

47 Umeda Y, Marui T, Matsuno Y, et al. Skeletal muscle targeting in vivo electroporation-mediated HGF gene therapy of bleomycin-induced pulmonary fibrosis in mice. Lab Invest 2004; 84: 836-844.

48 Gazdhar A, Fachinger P, van Leer C, et al. Gene transfer of hepatocyte growth factor by electroporation reduces bleomycin-induced lung fibrosis. Am J Physiol Lung Cell Mol Physiol 2007; 292: L529-L536.
49 Marshall RP. The pulmonary renin-angiotensin system. Curr Pharm Des 2003; 9: 715-722.

50 Nadrous HF, Ryu JH, Douglas WW, Decker PA, Olson EJ. Impact of angiotensin-converting enzyme inhibitors and statins on survival in idiopathic pulmonary fibrosis. Chest 2004; 126: 438-446.

51 Molteni A, Moulder JE, Cohen EF, et al. Control of radiation-induced pneumopathy and lung fibrosis by angiotensin-converting enzyme inhibitors and an angiotensin II type 1 receptor blocker. Int J Radiat Biol 2000; 76: 523-532.

52 Molteni A, Moulder JE, Cohen EP, et al. Prevention of radiation-induced nephropathy and fibrosis in a model of bone marrow transplant by an angiotensin II receptor blocker. Exp Biol Med 2001; 226: 1016-1023.

53 Molteni A, Wolfe LF, Ward WF, et al. Effect of an angiotensin II receptor blocker and two angiotensin converting enzyme inhibitors on transforming growth factor- $\beta$ (TGF- $\beta$ ) and $\alpha$-actomyosin ( $\alpha$-SMA), important mediators of radiation-induced pneumopathy and lung fibrosis. Curr Pharm Des 2007; 13: 1307-1316.

54 Uhal BD, Gidea C, Bargout R, et al. Captopril inhibits apoptosis in human lung epithelial cells: a potential antifibrotic mechanism. Am J Physiol Lung Cell Mol Physiol 1998; 275: L1013-1017.

55 Woo DS, Seol WJ, Kyung SY, et al. The therapeutic effect of angiotensin II receptor antagonist in idiopathic pulmonary fibrosis. Tuberc Respir Dis 2003; 55: 478-487. 